PROCEEDINGS OF THE

AMERICAN MATHEMATICAL SOCIETY

Volume 127, Number 12, Pages 3697-3706

S 0002-9939(99)05377-0

Article electronically published on August 5, 1999

\title{
TIGHT CONTACT STRUCTURES VIA DYNAMICS
}

\author{
JOHN ETNYRE AND ROBERT GHRIST
}

(Communicated by Ronald A. Fintushel)

\begin{abstract}
We consider the problem of realizing tight contact structures on closed orientable three-manifolds. By applying the theorems of Hofer et al., one may deduce tightness from dynamical properties of (Reeb) flows transverse to the contact structure. We detail how two classical constructions, Dehn surgery and branched covering, may be performed on dynamically-constrained links in such a way as to preserve a transverse tight contact structure.
\end{abstract}

\section{CONTACT GEOMETRY And DYNAMics}

For a more thorough treatment of the basic definitions and theorems related to the geometry and dynamics of contact structures see, e.g., [1].

A contact structure $\xi$ on a 3-manifold $M$ is a totally nonintegrable 2-plane field in $T M$. More specifically, at each point $p \in M$ we have a 2-plane $\xi_{p} \subset T_{p} M$ that varies smoothly with $p$, with the property that $\xi$ is nowhere integrable in the sense of Frobenius, i.e., there exists (locally) a defining 1-form $\alpha$ (whose kernel is $\xi$ ) such that $\alpha \wedge d \alpha \neq 0$. If $\alpha$ is globally defined, $\xi$ is called orientable and $\alpha$ a contact 1 -form for $\xi$. We adopt the common restriction to orientable contact structures.

The interesting (and difficult) problems in contact geometry are all of a global nature: Darboux's Theorem (see, e.g., $[23,1]$ ) implies that all contact structures are locally contactomorphic, or diffeomorphic preserving the plane fields. A similar result holds for a surface $\Sigma$ in a contact manifold $(M, \xi)$ as follows. Generically, $T_{p} \Sigma \cap \xi_{p}$ will be a line in $T_{p} \Sigma$. This line field integrates to a singular foliation $\Sigma_{\xi}$ called the characteristic foliation of $\Sigma$. One can show, as in the single-point case of Darboux's Theorem, that $\Sigma_{\xi}$ determines the germ of $\xi$ along $\Sigma$.

There has recently emerged a fundamental dichotomy in three dimensional contact geometry. A contact structure $\xi$ is overtwisted if there exists an embedded disk $D$ in $M$ whose characteristic foliation $D_{\xi}$ contains a limit cycle. If $\xi$ is not overtwisted, then it is called tight. Eliashberg [6] has completely classified overtwisted contact structures on closed 3-manifolds - the geometry of overtwisted contact structures reduces to the algebra of homotopy classes of plane fields. Such insight into tight contact structures is slow in coming. The only general method for constructing tight structures is by Stein fillings (see [14, 7]), and the uniqueness

Received by the editors January 28, 1998.

1991 Mathematics Subject Classification. Primary 53C15, 57M12; Secondary 58F05.

Key words and phrases. Tight contact structures, Reeb flows.

The first author was supported in part by NSF Grant \# DMS-9705949.

The second author was supported in part by NSF Grant \# DMS-9508846.

(C)1999 American Mathematical Society 
question has only been answered on $S^{3}[8], T^{3}[13,20]$, most $T^{2}$-bundles over $S^{1}$ [13], and certain lens spaces $L(p, q)[10]$.

Thus we have the fundamental open question: does every 3-manifold $M$ admit a tight contact structure? Martinet [22] and Thurston and Winkelnkemper [26] have used surgery techniques to show that all closed 3-manifolds admit contact structures. However, their constructions do not yield tight contact structures. The current state of affairs is to be found in a recent theorem of Eliashberg and Thurston [9], who show how to perturb taut foliations into tight contact structures. Then, the theorems of Gabai [12] imply that any closed orientable irreducible 3-manifold with nonzero second Betti number $\beta_{2}$ supports a tight contact structure.

The outline of this paper is as follows: the remainder of this section consists of dynamical preliminaries and a recollection of the striking work of Hofer et al. concerning Reeb fields. We proceed in $\S 2$ to carefully modify the well-known construction of Dehn surgery to preserve a tight contact structure when surgering over certain links. In $\S 3$, we turn to the procedure of branched covering and again show how to perform this construction in such a way as to preserve a tight contact structure. In both cases, the link that the surgery/branching is performed on is constrained by the associated dynamics; thus, unfortunately, only certain tight manifolds are obtained by our methods. In particular, we do not surpass the existing theorems of Eliashberg and Thurston. However, this marks the first example of proving tightness of surgered contact structures without means of Stein filling. It is unknown whether the structures we construct are Stein fillable in general.

1.1. The dynamics of Reeb flows. A contact 1-form $\alpha$ carries more geometry than does its contact structure $\xi=\operatorname{ker} \alpha$. In particular, given a contact form $\alpha$ there is a vector field $X$ uniquely determined by $\alpha(X)=1$ and $d \alpha(X, \cdot)=0$. The vector field $X$ is called the Reeb vector field [25], and it encapsulates the "extra geometry" $\alpha$ carries, since the Reeb field is characterized by the properties of being transverse to $\xi$ and preserving the 1-form $\alpha$. In his recent work on the Weinstein conjecture [17] Hofer has found deep connections between the dynamics of the Reeb vector field $X$ and the tightness of $\xi$ :

Theorem 1.1 (Hofer [17]). Let $\xi$ be an overtwisted contact structure on the closed 3-manifold $M$. Then the flow of the Reeb vector field associated to any contact 1-form generating $\xi$ has at least one closed orbit of finite order in $\pi_{1}(M)$.

This can be refined by considering the dynamics of the closed orbits. Following the standard usage [19], a periodic orbit in a Hamiltonian flow is either degenerate or nondegenerate, depending on whether the spectrum associated to the linearized return maps for the orbits contains, or excludes, respectively, one. The nondegenerate periodic orbits are either elliptic or hyperbolic, depending on whether these eigenvalues are on the unit circle or not, respectively.

Theorem 1.2 (Hofer, Wyzocki, and Zehnder [18]). Let $\xi$ be an overtwisted contact structure on the closed 3-manifold $M$. Then if the flow of the Reeb vector field associated to a contact 1-form generating $\xi$ has no degenerate periodic orbits, then there exists at least one closed hyperbolic orbit of finite order in $\pi_{1}(M)$.

The proofs of the above theorems are highly nontrivial, relying primarily on Gromov's theory of pseudoholomorphic curves [16]. 


\section{Dehn surgery on tight COntaCt StruCtures}

The operation of Dehn surgery is a very efficient way of constructing closed orientable 3-manifolds. A classical theorem in 3-manifold topology asserts that any closed orientable 3-manifold is obtainable via surgery on a link in $S^{3}$ [27, 21]. In this section, we show how to preserve tightness under certain circumstances.

2.1. Dehn surgery. The object of Dehn surgery on a 3-manifold is to drill out a solid torus and replace it with another solid torus inserted with "twists."

Let $\gamma$ denote a simple closed curve in $M^{3}$ having tubular neighborhood $N$ diffeomorphic to $D^{2} \times S^{1}$, with $\gamma$ as $\{0\} \times S^{1}$. Choose cylindrical coordinates $(\rho, \theta, \phi)$ on $N$ such that the boundary curves $m=\{(1, \theta, 0)\}$ and $\ell=\{(1,0, \phi)\}$ correspond respectively to a meridian and a longitude of $N$. The meridional curve is canonically defined, whereas the choice of the longitudinal curve depends on the framing of the coordinate system. Denote by $\Psi: \partial N \rightarrow \partial N$ the diffeomorphism

$$
\Psi\left(\begin{array}{c}
\theta \\
\phi
\end{array}\right)=\left[\begin{array}{ll}
p & s \\
q & t
\end{array}\right]\left(\begin{array}{c}
\theta \\
\phi
\end{array}\right)
$$

where $p, q, s$, and $t$ are integers satisfying $p t-q s=1$. The $p / q$ Dehn surgery of $M$ along $\gamma$ is performed by removing $N$ from $M$ and regluing it via $\Psi$,

$$
M_{\gamma}(p / q):=\overline{M \backslash N} \cup_{\Psi} N,
$$

resulting in the new manifold $M_{\gamma}(p / q)$ (completely determined by $\gamma, p$ and $q$ ).

2.2. Model contact structures on $S^{1} \times D^{2}$. When performing Dehn surgery we will need to keep track of the Reeb vector field in order to use Hofer's theorem to conclude that our surgered manifold is tight. This is done by constructing model contact forms on $S^{1} \times D^{2}$. To this end, for any fixed $r>0$ we define the Hamiltonian function $H(\mathbf{x}):=\left(x_{1}^{2}+y_{1}^{2}\right)+\frac{1}{r^{2}}\left(x_{2}^{2}+y_{2}^{2}\right)$ on $\mathbb{R}^{4}$, where $\mathbf{x}:=\left(x_{1}, y_{1}, x_{2}, y_{2}\right)$. The 1-form $\alpha:=\frac{1}{2}\left(x_{1} d y_{1}-y_{1} d x_{1}+x_{2} d y_{2}-y_{2} d x_{2}\right)$ restricts to a tight contact 1-form $\hat{\alpha}$ on $\hat{S}=H^{-1}(1)[3]$. We set $S:=\left\{\left(x_{1}, y_{1}, x_{2}, y_{2}\right) \mid x_{1}^{2}+y_{1}^{2}+x_{2}^{2}+y_{2}^{2}=1\right\}$ and define the map $\psi: S \rightarrow \hat{S}: \mathbf{x} \mapsto \mathbf{x} / \sqrt{H(\mathbf{x})}$. Thus we obtain the tight contact structure $\alpha_{H}:=\psi^{*}(\hat{\alpha})$ on $S$. One may easily check that $\alpha_{H}=\frac{1}{H(x)} \alpha$.

Choose coordinates $0 \leq \phi<2 \pi$ on $S^{1}$ and polar coordinates $(\rho, \theta)$ on $D^{2}$. In these coordinates we define a map

$$
f: S^{1} \times D^{2} \rightarrow S:(\phi,(\rho, \theta)) \mapsto\left(\sin \rho e^{i \theta}, \cos \rho e^{i \phi}\right) .
$$

We define our distinguished contact forms on $S^{1} \times D^{2}$ as $\alpha_{r}:=f^{*}\left(\alpha_{H}\right)$, which in the above coordinates is

$$
\alpha_{r}=\frac{1}{\left(\sin ^{2} \rho+\frac{1}{r^{2}} \cos ^{2} \rho\right)}\left(\sin ^{2} \rho d \theta+\cos ^{2} \rho d \phi\right) .
$$

It is now simple to check

1. For a fixed $\rho$ we get a torus $T_{\rho}$ in $S^{1} \times D^{2}$ by letting $\theta$ and $\phi$ vary. The characteristic foliation on $T_{\rho}$ is by lines with slope $-\tan ^{2} \rho$.

2. The Reeb vector field of $\alpha_{r}$ is tangent to the tori $T_{\rho}$ and has slope $\frac{1}{r^{2}}$ independent of $\rho$. 
2.3. Tight surgery. It is possible to perform Dehn surgery on a contact manifold and obtain a new contact manifold [22]; however, without great care the contact structure thus constructed will be overtwisted. Using Stein fillings, Eliashberg [7] and Gompf [14] have shown how to build tight contact structures by certain surgeries on any knot in Stein fillable 3-manifolds (actually symplectically semifillable would suffice, cf. [28]). In this section we show how to obtain a tight manifold by performing any surgery on a certain knot (e.g., the unknot in $S^{3}$ ).

Remark 2.1. To illustrate the difficulties in this type of construction consider the following situation originally described to the authors by Maker-Limanov. Let $\gamma$ be a closed transversal curve in $S^{3}$ (equipped with its standard contact structure). We can find a neighborhood of $\gamma$ that is contactomorphic to $S^{1} \times D^{2}$ with the contact structure $\operatorname{ker}\left(d \phi+\rho^{2} d \theta\right)$, where $\phi$ is the angle coordinate on $S^{1}$ and $(\rho, \theta)$ are polar coordinates on $D^{2}$. Suppose that this neighborhood is large enough to include the torus $T$ formed by setting $\rho=\sqrt{2}$. Now perform $-2 / 1$ Dehn surgery on $\gamma$ by cutting and pasting a solid torus not intersecting $T$. The characteristic foliation on $T$ is by $(-2,1)$ curves; thus they bound disks in the surgered manifold. Since the characteristic foliation of $T$ has no singularities, it is not hard to find a disk with one of these $(-2,1)$ curves as a limit cycle on its boundary. Thus the contact structure one obtains is overtwisted. When surgering tight contact structures one must be careful to perform surgery on sufficiently "large" tori.

Theorem 2.2. On $S^{3}$ with the (unique) tight contact structure, there exist tight $(p, q)$-Dehn surgeries on the unknot for arbitrary $p, q$.

Proof. For some irrational $r>0$, consider the contact form $\alpha_{H}$ on $S^{3}$ defined in $\S 2.2$. The Reeb field associated to $\alpha_{H}$ has precisely two periodic orbits, both of which are elliptic. On a neighborhood of each of these closed orbits, the contact form appears as in Equation (2.4); hence, the Reeb flow lies on invariant tori. We will remove an invariant neighborhood of one of the periodic orbits, $\gamma$, and glue in a solid torus using $\Psi$ (from Equation (2.1)), thus performing a $p / q$ Dehn surgery on the unknot $\gamma$.

Place coordinates $(\phi,(\rho, \theta))$ on $S^{1} \times D^{2}$ as in Section 2.2. Recall that for fixed $\rho$ the torus $T_{\rho}$ in $S^{1} \times D^{2}$ has as its characteristic foliation lines of slope $m_{\rho}=-\tan ^{2} \rho$, and the Reeb vector field is tangent to $T_{\rho}$ with slope $\frac{1}{r^{2}}$. Pulling $\alpha_{r}$ back by $\Psi$ we obtain a contact structure on a neighborhood of the boundary in $S^{1} \times D^{2}$ with characteristic foliation on each $T_{\rho}$ of slope

$$
n_{\rho}=-\left(\frac{p m_{\rho}-q}{s m_{\rho}-t}\right)
$$

and Reeb vector field tangent to $T_{\rho}$ with slope

$$
\bar{r}=-\left(\frac{p-q r^{2}}{s-t r^{2}}\right)
$$

It is easy to check that given $p$ and $q$, one can find $s, t, r$ and $\rho \in\left[0, \frac{\pi}{2}\right)$ such that $p t-q s=1, \bar{r}>0$, and $n_{\rho}<0$. Now let $N=S^{1} \times D^{2}$, where the $\rho$ variable is restricted to lie in the interval $\left[0, \tan ^{-1} \sqrt{\left(n_{\rho}\right)}\right]$, and let $\alpha_{1 / \sqrt{r}}$ be the model contact form constructed in $\S 2.2$. One can now construct a map $\Phi$ from a neighborhood of the boundary of $\left(N, \alpha_{1 / \sqrt{\bar{r}}}\right)$ to a neighborhood of the boundary of $\left(S^{1} \times D^{2}, \Psi^{*} \alpha_{r}\right)$ that preserves the contact form (to arrange this, make the map the identity on 
the invariant tori and reparametrize in the $\rho$ direction so that the characteristic foliations on tori and the direction of the Reeb vector field are preserved). One may then use $\Psi \circ \Phi$ to glue $\left(N, \alpha_{1 / \sqrt{\bar{r}}}\right)$ to $\left(S^{3} \backslash\left(S^{1} \times D^{2}\right), \alpha_{r}\right)$. This contact form has a Reeb field with neither degenerate nor hyperbolic periodic orbits; hence it is tight by Theorem 1.1.

Remark 2.3. Recall that the lens space $L(p, q)$ is obtained from $S^{3}$ by performing $-p / q$ Dehn surgery on an unknot. It has been known for a long time that all lens spaces admit tight contact structures. Our interest in this theorem is the novel way of proving that these contact structures are tight - using dynamical properties of the Reeb vector fields to detect subtle geometric information. This is the first "surgery" construction of tight contact structures that does not rely on Stein filling.

Remark 2.4. It is not hard to compute the Euler class of the contact structure $\xi$ constructed in this example: let $D$ be the 2-skeleton of the natural CW-decomposition coming from the surgery (which follows since we are surgering an unknot). Then, using as the generator of $H^{2}(L(p, q) ; \mathbb{Z})$ the cochain that evaluates to one on $D$, the Euler class of $\xi$ is $e(\xi)=q+1$. This follows from the formula for the Euler class in [10], given that the characteristic foliation on $D$ has exactly one singular point (as can be seen using the local models). When $p$ is even, there is a refinement of the Euler class defined in [14] which may be likewise computed (see [10] for a precise statement). We note that in every case considered, this Euler class can be realized by a tight contact structure that can be Stein filled - it is unknown whether this is true in general.

Remark 2.5. In the above proof we never specifically addressed the problem of surgering on "sufficiently large tori" discussed in Remark 2.1. It is an interesting exercise to see that if one chooses $s, t, r$ and $\rho$ so that $\bar{r}>0$ and $n_{\rho}<0$, then a sufficiently large torus is being surgered.

\section{BRANCHED COVERS AND Tight CONTACT STRUCTURES}

Another way of building all 3-manifolds is via branched covers over knots and links. In this section, we show how one may perform tight branched coverings of 3-manifolds along closed orbits of a suitable Reeb flow.

3.1. Branched covers over links. To branch over a knot, one removes a neighborhood of the knot, takes an $n$-fold cover of the complement, and then fills in the tube(s) in such a way that the cross-sectional map in the meridional direction is the $m$-fold singular cover of the disc $D \subset \mathbb{C}$ given by $z \mapsto z^{m}$. More specifically, let $\gamma:=\left\{\gamma_{i}\right\}_{1}^{n}$ denote an $n$-component link in $M^{3}$. Denote by $N$ a tubular neighborhood of $\gamma$ and by $E:=\overline{M \backslash N}$ the exterior of $N$. For any subgroup $G<\pi_{1}(E)$ of finite index, there is a well-defined compact cover $p: M_{G} \rightarrow E$. Denote by $\left\{T_{i}\right\}$ the collection of boundary components of $M_{G}$, each diffeomorphic to a torus. The cover $p$ restricts to $p_{i}:=\left.p\right|_{T_{i}}$ on each torus.

Each boundary component of $E$ is a torus which may be fitted with a meridian in such a way that each $p_{i}$ lifts this to a meridian for $T_{i}$ via an $m_{i}$-fold cover. We may then construct $\tilde{M}$ by filling in the $T_{i}$ 's with $S^{1} \times D^{2}$ 's, sending $\{$ pt. $\} \times \partial D^{2}$ to the meridian. After choosing a longitude for all the tori, each $p_{i}$ can be represented as $p_{i}(\theta, \phi)=(m \theta+k \phi, l \phi)$, where $\theta$ and $\phi$ are the meridional and longitudinal coordinates, respectively, and $m=m_{i}, k$, and $l$ are integers. If we extend each 
$p_{i}: T^{2} \rightarrow T^{2}$ to a map $\overline{p_{i}}: D^{2} \times S^{1} \rightarrow D^{2} \times S^{1}$ via $(\rho, \theta, \phi) \mapsto(\rho, m \theta+k \phi, l \phi)$, then the branched cover of $M$ over $\gamma$ via $G$ is defined to be $\tilde{M}$ with projection

$$
\tilde{p}: \tilde{M} \rightarrow M\left\{\begin{array}{l}
p \text { on } M_{G}, \\
\overline{p_{i}} \text { on each } D^{2} \times S^{1} .
\end{array}\right.
$$

Note the above projection map is not a smooth map since the $\overline{p_{i}}$ are not smooth at $\rho=0$. One could also define the $\overline{p_{i}}$ 's so that they are smooth: $(\rho, \theta, \phi) \mapsto$ $\left(\rho^{2}, m \theta+k \phi, l \phi\right)$. In this case, however, $d \overline{p_{i}}=0$ at $\rho=0$. We will make use of both the smooth and nonsmooth versions in the following section.

3.2. Tight branching over elliptic orbits. In [15], Gonzalo demonstrates lifting contact structures via a branched covering, and in this way also constructs contact structures on all closed orientable 3-manifolds. There is no indication of tightness of such structures. In general, taking the (unbranched) cover of a tight contact manifold can yield overtwisted contact manifolds [14] — so much more so for branched coverings.

We begin by showing how one can branch over certain elliptic periodic orbits in a Reeb field to obtain tight contact structures. We say a periodic orbit $\gamma$ in the Reeb flow of a contact form $\alpha$ is locally integrable at $\gamma$ if there exist a neighborhood $N$ of $\gamma$ and (smooth) coordinates $(\rho, \theta, \phi)$ such that the Reeb field takes the form $a(\rho) \frac{\partial}{\partial \theta}+b(\rho) \frac{\partial}{\partial \phi}$. These are precisely the action-angle coordinates from an integrable two degree-of-freedom Hamiltonian system, restricted to an energy surface.

Theorem 3.1. Let $\alpha$ be a contact 1-form on $(M, \xi)$ such that the associated Reeb vector field $X$ either (1) supports no closed orbits of finite order in $\pi_{1}(M)$; or (2) supports no degenerate orbits and no hyperbolic orbits of finite order in $\pi_{1}(M)$. Moreover, assume that $X$ admits a link of locally integrable periodic orbits $\gamma$. Then any branched cover $\tilde{p}: \tilde{M} \rightarrow M$ over $\gamma$ has a tight contact structure $\xi^{p}$, which is the lift of $\xi$ outside of a neighborhood of $\gamma$.

Proof. We assume without loss of generality that $\gamma$ is a single-component link. In order to pull back the form $\alpha$ to a smooth contact form on the branched cover, we need to ensure that the form can be made locally $\theta$-equivariant.

Case 1: If the Reeb field $X$ has degenerate periodic orbits near $\gamma$, then we may perturb the contact form as follows. Near any transverse loop such as $\gamma$, there exist coordinates for which the contact structure is the kernel of $d \phi+\rho^{2} d \theta[1$, Thm. 8.3]. In these coordinates, $\alpha$ is of the form $f(\rho, \theta, \phi)\left(d \phi+\rho^{2} d \theta\right)$ for some positive function $f$. To remove the $\theta$-dependence of $\alpha$ near $\gamma$, Taylor-expand $f$ as $f=f_{0}(\rho, \phi)+\bar{f}(\rho, \theta, \phi)$, where $\bar{f}$ is $O(\rho)$. Then, choose a bump function $\chi(\rho)$ with support on $N$ attaining the value 1 on a very small neighborhood of $\rho=0$, and consider the form $\beta:=g(\rho, \theta, \phi)\left(d \phi+\rho^{2} d \theta\right)$, where $g:=\left(f_{0}+(1-\chi) \bar{f}\right)$. Since $\bar{f}$ is $O(\rho)$, the fact that $f>0$ implies that $g>0$, and, hence, that $\beta$ is contact.

The Reeb field $Y$ for $\beta$ may have a very different periodic orbit structure from $X$. Since $\gamma$ is locally integrable, the orbits of $Y$ are bound by invariant tori outside of a very small neighborhood. Hence, every closed orbit of $Y$ near $\gamma$ is a multiple of $\gamma$ in $\pi_{1}(M)$. It follows from hypothesis that $\gamma$ is of infinite order in $\pi_{1}(M)$, so it suffices to show that this multiple is always nonzero. To do this, note that the $\frac{\partial}{\partial \phi}$ component of $Y$ is given by $\left(2 g+\rho g_{\rho}\right) / 2 g^{2}$. It suffices to show that the numerator is nonzero on $N$. The first term, $2 g$, is strictly positive. The second term, $\rho g_{\rho}$, may be made small through the choice of neighborhood and $\chi$, and hence does not 
overpower the (nonzero) $2 g$ term. Thus every periodic orbit of $Y$ is also of infinite order in $\pi_{1}$.

We may now branch since $\beta$ has the local normal form

$$
\beta=f_{0}(\rho, \phi)\left(\rho^{2} d \theta+d \phi\right)
$$

Pulling $\beta$ back by the nonsmooth covering map $\overline{p_{i}}$ yields the local form

$$
\tilde{\beta}=m f_{0}(\rho, l \phi) \rho^{2} d \theta+\left(k f_{0}(\rho, l \phi) \rho^{2}+l f_{0}(\rho, l \phi)\right) d \phi
$$

on the branched cover. This form clearly extends over $\rho=0$, since $\beta$ was a smooth form. Thus $\tilde{\beta}$ is a well-defined 1 -form on $\tilde{N}$, which is a contact form since $\tilde{\beta} \wedge$ $d \tilde{\beta}=\left(2 m l f_{0}^{2}\right) \rho d \rho \wedge d \theta \wedge d \phi$. Moreover, since finite-order closed orbits on $\tilde{M}$ must project to finite-order closed orbits on $M$, the Reeb field $Y$ of $\tilde{\beta}$ on $\tilde{M}$ satisfies the hypotheses of Theorem 1.1, and the contact structure $\xi^{p}=\operatorname{ker}(\tilde{\alpha})$ is tight.

Case 2: If, in contrast, there are no degenerate periodic orbits near $\gamma$, then we may not perturb $\alpha$ to induce such. However, using the action-angle coordinates, we have that $X=a(\rho) \frac{\partial}{\partial \theta}+b(\rho) \frac{\partial}{\partial \phi}$. Since there are no degenerate periodic orbits, $a$ and $b$ are irrationally-related constants. In these coordinates, $\alpha$ takes on the form $\alpha=f d \theta+g d \phi+h d \rho$, where all the coefficients $f, g$, and $h$ are functions of all three coordinates.

By the definition of $X$, one has that $a f+b g=1$ and $a\left(f_{\rho}-h_{\phi}\right)+b\left(g_{\rho}-h_{\phi}\right)=0$. By differentiating the former with respect to each variable, one can derive the equations $f_{\phi}=-\frac{a}{b} f_{\theta}, g_{\phi}=-\frac{a}{b} g_{\theta}$, and $h_{\phi}=-\frac{a}{b} h_{\theta}$. These define first-order PDEs on $N$, and, in particular, on the invariant tori which foliate $N$. It is clear that the solutions to the Reeb field are characteristics of the PDEs; however, these are dense on the invariant tori since $X$ has no degenerate closed orbits. Hence, $f, g$, and $h$ are constants on each torus $\rho=c$ and these are all functions of $\rho$.

We thus have $\alpha$ of the form

$$
\alpha=f(\rho) d \theta+g(\rho) d \phi+h(\rho) d \rho .
$$

Pulling $\alpha$ back by the nonsmooth covering map $\overline{p_{i}}$ yields the local form

$$
\tilde{\alpha}=m f(\rho) d \theta+(k f(\rho)+l g(\rho)) d \phi+h(\rho) d \rho
$$

on the branched cover. This form clearly extends over $\rho=0$, since $\alpha$ is a smooth form. Thus $\tilde{\alpha}$ is a well-defined 1 -form on $\tilde{N}$ which is a contact form since $\tilde{\alpha} \wedge d \tilde{\alpha}=$ $m l \alpha \wedge d \alpha$. Hence $\tilde{p}^{*} \alpha$ extends to a contact form on $\tilde{M}$. Moreover, since hyperbolic closed orbits on $\tilde{M}$ must project to (infinite-order) hyperbolic closed orbits on $M$, the Reeb field of $\tilde{\alpha}$ on $\tilde{M}$ satisfies the hypotheses of Theorem 1.2 and the contact structure $\xi^{p}=\operatorname{ker}(\tilde{\alpha})$ is tight.

Example 3.2 (lens spaces). Consider the lens space $L(p, q)$ with the contact form $\alpha$ as constructed in Theorem 2.2. The Reeb vector field is an integrable field with precisely two closed orbits, $\gamma_{1}$ and $\gamma_{2}$, which form the cores of a genus one Heegaard decomposition. As these orbits are elliptic, we may apply Case 2 of Theorem 3.1. The covers of $L(p, q)$ branched over $\gamma_{1} \cup \gamma_{2}$ are of the form $L\left(p, q^{\prime}\right)$ : it is an instructive exercise to determine $q^{\prime}$ for $p, q$ and the branching data. It would appear that we have found more tight contact structures on $L\left(p, q^{\prime}\right)$; however, it can be demonstrated that these structures are all contactomorphic to the one constructed in Theorem 2.2 (compute the Euler classes and then appeal to the classification in [10]). 
Example 3.3 (the three-torus). The contact form

$$
\alpha=(\sin z d x+\cos z d y)+\frac{1}{2}(\sin x d y+\cos x d z)
$$

has as its Reeb field (up to a nonzero rescaling)

$$
X=2 \sin z \frac{\partial}{\partial x}+(\sin x+2 \cos z) \frac{\partial}{\partial y}+\cos x \frac{\partial}{\partial z} .
$$

This flow arises in the study of steady inviscid fluid flows [5]. As this is a level set of an integrable Hamiltonian flow, it is simple to check that this vector field on $T^{3}$ has no contractible closed orbits. The elliptic integral curves $\{(\pi / 2, y, 0): y \in \mathbb{R} / \mathbb{Z}\}$ and $\{(-\pi / 2, y, \pi): y \in \mathbb{R} / \mathbb{Z}\}$ are each a generator of $H_{1}\left(T^{3}\right)$ in the standard basis and are locally integrable orbits. By Theorem 3.1 branching over these curves yields tight contact structures on surface bundles over $S^{1}$.

3.3. Tight branching over hyperbolic orbits. We now consider branching over hyperbolic orbits. This is a little more delicate, and we need to make stronger global assumptions on the flow.

Theorem 3.4. Let $\alpha$ be a contact 1-form on $M$ such that the associated Reeb vector field $X$ generates a structurally stable flow having no finite-order closed orbits (e.g., an Anosov flow). Let $\gamma$ be any link of periodic orbits in the flow of $X$, and $\tilde{M}$ any branched cover over $\gamma$. Then $\tilde{M}$ has a tight contact form which is the lift of $\alpha$ (outside of an arbitrarily small neighborhood of $\gamma$ ).

Proof. Consider a neighborhood $N$ of a component $\gamma_{i}$ of $\gamma$. Using the smooth branching map pull back $\left.\alpha\right|_{N}$ to a 1 -form $\beta$ on the cover $\tilde{N}$. This smooth form $\beta$ is a contact form off of $\rho=0$. Now set $\bar{\alpha}:=\beta+\epsilon u(\rho) \rho^{2} d \theta$, where $u(\rho)$ is a bump function with support on $\tilde{N}$ attaining 1 near $\rho=0$, and $\epsilon$ is a small constant. It is not hard to check that for small $\epsilon$ the form $\bar{\alpha}$ is a contact form on all of $N$. Note that $\rho=0$ is still a periodic orbit of the Reeb field $\bar{X}$ for $\bar{\alpha}$.

The perturbation to the contact form, and hence the Reeb field, is equivariant with respect to the branching map. Thus, away from $\rho=0$, the Reeb field of $\bar{\alpha}$ pushes down to a perturbation of the Reeb field of $\alpha$. Thus flow lines of the Reeb field of $\bar{\alpha}$ are mapped to flow lines of the perturbed field downstairs. Moreover, since the Reeb field downstairs is structurally stable, the perturbed field also has no contractible orbits, implying the same for the Reeb flow of $\bar{\alpha}$.

Example 3.5 (pseudo-Anosov Reeb fields). Let $M$ be the unit tangent bundle of a surface $\Sigma$ having constant negative curvature. The geodesic flow on $M$ is Anosov [2], and preserves a transverse contact structure [24]. Let $\alpha$ denote the natural contact form for which the flow is Reeb. We may apply Theorem 3.4 to $(M, \alpha)$ to conclude: arbitrary branched covers over closed geodesics yield tight contact manifolds. This construction gives many interesting manifolds.

Remark 3.6. The dynamics on the branched covers are no longer Anosov, but can be lifted so as to be pseudo-Anosov (see, e.g., [11]). This provides a curious set of examples in light of the recent work of Benoist et al., who show in [4] a strong rigidity among manifolds which admit an Anosov Reeb field. Namely, a Reeb field which is Anosov with $C^{\infty}$ splitting must be either a geodesic flow on a surface of constant negative curvature, or a certain time-reparametrization of this flow, or the lifted flow on an unbranched covering space of the unit tangent bundle. Our construction 
shows that relaxing the Anosov condition to a pseudo-Anosov condition greatly enlarges the class of 3-manifolds which admit such contact-preserving flows. This presents an interesting problem in itself: Classify which closed 3-manifolds admit a pseudo-Anosov Reeb flow with $C^{\infty}$ splitting.

\section{REFERENCES}

[1] B. Aebisher, Symplectic geometry, Progress in Math., no. 124, Birkhaüser, Berlin, 1994.

[2] D. Anosov, Geodesic flows on closed Riemannian manifolds with negative curvature, Proc. Skelkov Inst. 90 (1967). MR 39:3527

[3] D. Bennequin, Entrelacements et équations de Pfaff, Asterisque 107-108 (1983), 87-161. MR 86e: 58070

[4] Y. Benoist, P. Foulon, and F. Labourie, Flots d'Anosov à distributions stable et instable différentiables, J. Amer. Math. Soc. 5 (1992), no. 1, 33-74. MR 93b:58112

[5] T. Dombre, U. Frisch, J. Greene, M. Hénon, A. Mehr, and A. Soward, Chaotic streamlines in the ABC flows, J. Fluid Mech. 167 (1986), 353-391. MR 88f:76012

[6] Y. Eliashberg, Classification of overtwisted contact structures on 3-manifolds, Invent. Math. 98 (1989), 623-637. MR 90k:53064

[7] _ Topological characterization of Stein manifolds of dimension > 2, Int. J. of Math. 1 (1990), 29-46. MR 91k:32012

[8] , Contact 3-manifolds twenty years since J. Martinet's work, Ann. Inst. Fourier, Grenoble 42(1-2) (1992), 165-192. MR 93k:57029

[9] Y. Eliashberg and W. Thurston, Confoliations, University Lecture Series, vol. 13, American Mathematical Society, 1998. MR 98m:53042

[10] J. Etnyre, Tight contact structures on lens spaces, Preprint, 1997.

[11] A. Fathi, F. Laudenbach, and V. Poenaru et al., Travaux de Thurston sur les surfaces, Astérique 66-67 (1979).

[12] D. Gabai, Foliations and the topology of 3-manifolds I, J. Diff. Geom. 18 (1983), 445-503. MR 86a:57009

[13] E. Giroux, Une structure de contact, même tendue est plus ou moins tordue, Ann. Scient. Ec. Norm. Sup. 27 (1994), 697-705. MR 96b:57034

[14] R. Gompf, Handlebody construction of Stein surfaces, Preprint, 1996.

[15] J. Gonzalo, Branched covers and contact structures, Proc. Am. Math. Soc. 101 (1987), 347352. MR 88k:53058

[16] M. Gromov, Pseudoholomorphic curves in symplectic manifolds, Invent. Math. 82 (1985), 307-347. MR 87j:53053

[17] H. Hofer, Pseudoholomorphic curves in symplectizations with applications to the Weinstein conjecture in dimension three, Invent. Math. 114 (1993), 515-563. MR 94j:58064

[18] H. Hofer and M. Kriener, Holomorphic curves in contact dynamics, Notes from 1997 Park City Mathematics Institute, July 1997.

[19] H. Hofer and E. Zehnder, Hamiltonian dynamics and symplectic invariants, Birkhäuser, Berlin, 1994. MR 96g:58001

[20] Y. Kanda, The classification of tight contact structures on the 3-torus, Comm. in Anal. and Geom. 5 (1997), 413-438. MR 99c:57054

[21] W. B. R. Lickorish, A representation of orientable combinatorial 3-manifolds, Ann. of Math. 76 (1962), 531-538. MR 27:1929

[22] J. Martinet, Forms de contact sur les variétés de dimension 3, Springer Lecture Notes in Math., no. 209, 142-163, Springer Lecture Notes in Math., no. 209, Springer-Verlag, 1971, pp. 142-163. MR 50:3263

[23] D. McDuff and D. Salamon, Introduction to symplectic topology, Oxford University Press, New York, 1995. MR 97b:58062

[24] J. Plante, Anosov flows, Am. J. Math. 94 (1972), 729-754. MR 51:14099

[25] G. Reeb, Sur certaines propriétés topologiques des trajectoires des systèmes dynamiques, Acad. Roy. Belgique Cl. Sci. Mém. Coll. 27(9) (1952), 1-64. MR 15:336b

[26] W. Thurston and H. Winkelnkemper, On the existence of contact forms, Proc. Am. Math. Soc. 52 (1975), 345-347. MR 51:11561 
[27] A. Wallace, Modifications and cobounding manifolds, Can. J. Math. 12 (1960), 503-528. MR 23:A2887

[28] A. Weinstein, Contact surgery and symplectic handlebodies, Hokkaido Math. J. 20 (1991), 241-251. MR 92g:53028

Department of Mathematics, Stanford University, Palo Alto, California 94305

E-mail address: etnyre@math.stanford.edu

School of Mathematics, Georgia Institute of Technology, Atlanta, Georgia 30332

E-mail address: ghrist@math.gatech.edu 